\title{
Heme oxygenase/carbon monoxide pathway inhibition plays a role in ameliorating fibrosis following splenectomy
}

\author{
QIU-MING WANG ${ }^{1}$, ZHI-JUN DUAN $^{1}$, JIAN-LING DU $^{2}$, SHI-BIN GUO $^{1}$, XIAO-YU SUN $^{1}$ and ZHEN LIU ${ }^{1}$ \\ Departments of ${ }^{1}$ Gastroenterology and ${ }^{2}$ Endocrinology, The First Affiliated Hospital of \\ Dalian Medical University, Dalian, Liaoning 116011, P.R. China
}

Received December 21, 2012; Accepted February 22, 2013

DOI: $10.3892 /$ ijmm.2013.1309

\begin{abstract}
Splenectomy is a recognized therapy for liver cirrhosis with splenomegaly, since it decreases free iron concentration that accompanies the destruction of red blood cells. Heme oxygenase (HO)-1 and its by-products, iron and carbon monoxide (CO), play crucial roles in hepatic fibrosis. The aim of the present study was to determine whether splenectomy in cirrhotic rats induced by bile duct ligation (BDL), through the $\mathrm{HO} / \mathrm{CO}$ pathway, could slow down the development of liver fibrosis. Male Sprague-Dawley rats were divided randomly into the sham, BDL, splenectomy, Fe, zinc protoporphyrin ( $\mathrm{ZnPP}$ ) and cobalt protoporphyrin (CoPP) treatment groups, for inhibiting and inducing HO-1 expression. The level of HO-1 was detected by western blot analysis and reverse transcription-polymerase chain reaction. Serum carboxyhemoglobin $(\mathrm{COHb})$, iron and portal vein pressure (PVP) were also quantified. Liver iron was measured by atomic absorption spectrometry with acetylene-air flame atomization. HO- 1 and $\alpha$-smooth muscle actin ( $\alpha$-SMA) were localized by immunohistochemistry. Liver and spleen iron were visualized by Perls' Prussian blue staining. Hepatic fibrosis was assessed using hematoxylin and eosin (H\&E) staining. Enzyme-linked immunosorbent assay (ELISA) was used to detect serum transforming growth factor- $\beta 1$ (TGF- $\beta 1$ ). The results showed that liver, spleen and serum levels of $\mathrm{HO}-1, \mathrm{COHb}$ and iron were greatly enhanced in the BDL group compared with the sham group; they were reduced following splenectomy and ZnPP treatment, but were elevated in the CoPP and $\mathrm{Fe}$ groups. Hydroxyproline, TGF- $\beta 1, \alpha-S M A$, PVP and malonaldehyde levels were lower in the splenectomy and ZnPP groups compared to BDL, while higher levels were observed in the $\mathrm{CoPP}$ and Fe-treated groups. Our study shows that splenectomy reduces iron and $\mathrm{CO}$ levels in part by reducing $\mathrm{HO}-1$ expression, and it decreases portal pressure and slightly decreases hepatic fibroproliferation.
\end{abstract}

Correspondence to: Professor Zhi-Jun Duan, Department of Gastroenterology, The First Affiliated Hospital of Dalian Medical University, 222 Zhongshan Road, Dalian, Liaoning 116011, P.R. China E-mail: cathydoctor@sina.com

Key words: splenectomy, heme oxygenase-1, iron accumulation, portal vein pressure, bile duct ligation

\section{Introduction}

The spleen is connected to the other abdominal organs by the portal system. Portal hypertension and splenomegaly are often found in end-stage hepatic cirrhosis. As a treatment option, splenectomy is more widely accepted for patients without a donor suitable for liver transplantation (1). It is reported that splenectomy has beneficial effects on ameliorating reperfusion injury $(2,3)$ and that it also induces a significant reduction in portal system pressure, improving hepatic function $(4,5)$. Splenectomy also improves hepatic microcirculation, decreases spleen-derived endothelin-1, activates eNOS signaling, and inhibits the Rho-kinase pathway in rats with secondary biliary cirrhosis (6). Splenectomy also ameliorates liver injury in rats by mediating heme oxygenase-1 (HO-1) induction, thus yielding beneficial effects of massive hepatectomy and ischemia/reperfusion $(7,8)$. The mechanism by which splenectomy influences cirrhotic liver remains unclear.

Previous studies, including our own, show that the HO/carbon monoxide $(\mathrm{CO})$ pathway is involved in hepatic fibrosis and contributes to the hyperdynamic circulatory syndrome $(9,10)$. $\mathrm{HO}-1$ catalyses the oxidative degradation of heme to $\mathrm{CO}$, free iron, and biliverdin (11). CO, a gaseous messenger similar to $\mathrm{NO}$, leads to the production of cGMP, which mediates vasodilation (12). Our previous studies indicated that the $\mathrm{HO} / \mathrm{CO}$ pathway may contribute to renal and pulmonary vasodilation in cirrhotic rats induced by bile duct ligation (BDL) $(13,14)$. Whether splenectomy portal vein pressure (PVP) decreases are associated with a change in the $\mathrm{HO} / \mathrm{CO}$ pathway is not clear.

Cirrhotic patients often have hypersplenism due to splenomegaly. Therefore, leukopenia, thrombocytopenia, and anemia are major concerns in cirrhotic patients (15). Damaged erythrocytes may lead to iron overload as each unit of erythrocytes contains approximately $250 \mathrm{mg}$ of iron, thus increasing the risk of iron accumulation derived from increased heme degradation. Iron is an essential nutrient for growth and survival, but excess liver iron in particular induces oxidative stress and hepatic fibrogenesis (16). Iron is primarily accumulated in reticuloendothelial cells. The spleen also recycles iron stored in bone marrow and is essential for the removal of stored blood cells, and certain immune functions. Whether and how splenectomy influences iron homeostasis remains unclear.

Given the above-mentioned issues, the current study sought to evaluate how splenectomy impacts iron and CO levels, in 
part by reducing HO-1 expression, which further decreased PVP and reduced hepatic fibroproliferation.

\section{Materials and methods}

Animal care. The Animal Care and Use Committee of Dalian Medical University (Liaoning, China) approved the experimental protocols in accordance with guidelines established by the China Council on Animal Care.

Bile duct ligation and treatment of rats. The fifty healthy male SD rats, weighing 200-220 g, were obtained from the Laboratory Animal Center of Dalian Medical University and were randomly divided into six treatment groups: sham $(n=6)$, BDL $(n=10)$, cobalt protoporphyrin (CoPP) $(n=10)$, zinc protoporphyrin $(\mathrm{ZnPP})(\mathrm{n}=8)$, iron-dextran $(\mathrm{Fe})(\mathrm{n}=8)$ and splenectomy $(\mathrm{n}=8)$. They were housed in a specific pathogen free (SPF) center at a room temperature of $24-26^{\circ} \mathrm{C}$ and relative humidity of $60-65 \%$. Water was given ad libitum.

The rats were fed and housed for three days prior to any experimental protocols. Biliary cirrhosis was induced by $\operatorname{BDL}(17,18)$. Five groups underwent BDL together with a sham-operated animal group as a control. The Animal Care and Use Committee of Dalian Medical University approved all surgical procedures. Laparotomy was performed under anesthesia with ether. The bile duct was isolated and doubleligated with a 3-0 silk suture. The abdominal wall and skin were closed with a 4-0 silk suture, and antibiotic Gentamicin $(0.3 \mathrm{ml})$ was injected intramuscularly. The sham group underwent laparotomy with the bile duct isolated but not ligated. Two weeks after surgery, sham and BDL rats received a saline intraperitoneal injection. For the splenectomy group, the abdomen was opened through a midline incision, and the spleen was immobilized to the centre of the operative field after dissecting the surrounding ligaments. The hilar vessels were ligated with a 3-0 silk suture. The spleen was removed and the abdominal incision was closed. Other groups received an intraperitoneal injection of CoPP, $\mathrm{ZnPP}$, or iron-dextran (5, 5 and $50 \mathrm{mg} / \mathrm{kg}$ body weight), three times a week, respectively. Following establishment of the rat models, the number of rats was reduced to six in each group due to death in some groups.

ZnPP and CoPP (Sigma, St. Louis, MO, USA) were dissolved in $0.2 \mathrm{~mol} / 1$ of $\mathrm{NaOH}$, adjusted to $\mathrm{pH} 7.4$ and diluted in $0.85 \% \mathrm{NaCl}$ to $1 \mathrm{mg} / \mathrm{ml}$ as previously described, and used to inhibit and induce HO-1 expression. Fe (Sigma) was diluted in $0.85 \% \mathrm{NaCl}$ to $20 \mathrm{mg} / \mathrm{ml}$. Histostain ${ }^{\mathrm{TM}}$-Plus (SP9001) (Zhongshan Golden Bridge Biotechnology, Beijing, China), hydroxyproline (HYP), malonaldehyde (MDA) and glutathione (GSH) kits (Nanjing KeyGen Biotech Co. Ltd., China), transforming growth factor- $\beta 1$ (TGF- $\beta 1$ ) enzymelinked immunosorbent assay (ELISA) kit (HopeYear Medical Products Co., Ltd., China), and Takara RNA polymerase chain reaction (PCR) kits (AMV) version 3.0 (Takara Bio, Inc., Dalian, China) were used in this study.

Sample collection and examination. Two weeks after treatment, a catheter connected to a Pressure Transducer (BL-420F biological experimental system; Chengdu Technology and Market Co., Ltd., China), was placed in the portal vein to measure PVP. Subsequently, $1 \mathrm{ml}$ of arterial blood was withdrawn to measure carboxyhemoglobin $(\mathrm{COHb})$ using a RapidLab 1245 Blood Gas Analyzer (Siemens, New York, NY, USA). Levels of alanine aminotransferase (ALT), aspartate aminotransferase (AST), total bilirubin (TBIL), and serum iron were detected using a Hitachi 7600-110 Automatic Biochemical Analyzer (Hitachi Co., Tokyo, Japan). Levels of MDA and GSH were determined by a UV-2100 Spectrophotometer (Chemito Instruments Pvt., Ltd., Mumbai, India).

Serum TGF- $\beta 1$. TGF- $\beta 1$ expression was determined by ELISA at $450 \mathrm{~nm}$. Serum samples were assayed using 96-well microtiter plates coated with a polyclonal antibody against TGF- $\beta 1$.

Liver iron. Liver iron was determined by atomic absorption spectrometry with acetylene-air flame atomization. Analysis was performed using a Varian atomic absorption spectrometer (Mulgrave, Victoria, Australia) with a deuterium background correction. Measurements were performed with the analytical $248.3 \mathrm{~nm}$ line at a spectral interval of $0.2 \mathrm{~nm}$. Iron concentration was determined by the standard addition method. Samples were digested in an MDS 2000 microwave sample preparation system (CEM) in Teflon cartridges using a mixture of nitric acid $(5 \mathrm{ml})$ and hydrogen peroxide ( $2 \mathrm{ml}$ ) (ultrapure grade; both from Merck $\mathrm{KGa}$, Darmstadt, Germany) for $20 \mathrm{~min}$ at the 120 psi. Resulting products were analyzed directly in the Teflon cartridges.

Histology and immunohistochemistry. Part of the liver and spleen lobe were excised, fixed in $10 \%$ neutral formalin solution, and embedded in paraffin. Hematoxylin and eosin (H\&E) staining and Van Gieson's (VG) staining were performed according to standard procedures. Lesion severity was graded according to the methods described previously (19). Briefly, tissue sections $(4 \mu \mathrm{m})$ were treated with $\mathrm{HCl}(5 \%)$ to liberate ferric ions. Samples were then treated with $5 \%$ potassium ferrocyanide to produce insoluble ferric ferrocyanide. Slides were counterstained with neutral red. For immunohistochemical examination, deparaffinized sections were incubated with HO-1 antibodies (1:1,000 dilution; Abcam, Cambridge, MA, USA), or anti- $\alpha$-smooth muscle actin ( $\alpha$-SMA) antibody (1:100 dilution; Boster Biological Technology Ltd., Wuhan, China) and biotinylated secondary antibodies, followed by avidin-biotin-peroxidase complex. Images were analyzed by Image-Pro-Plus 6.0 software (Media Cybernetics, Rockville, MD, USA) to calculate area and mean density of positive expression. Statistical results from five visual fields were averaged from each sample.

Hepatic hydroxyproline content. Liver tissue $(100 \mathrm{mg})$ was prepared for HYP determination according to a modification of the kit method described. The HYP content of the liver served as an indirect measure of tissue collagen content and was expressed as microgram/gram of wet weight $(\mu \mathrm{g} / \mathrm{g})$.

Western blot analysis. The resected liver tissues were extracted with lysis buffer ( $1 \%$ Triton X-100; $50 \mathrm{mmol} / 1$ Tris- $\mathrm{HCl}$, $\mathrm{pH} 7.6 ; 150 \mathrm{mmol} / 1 \mathrm{NaCl}$; and $1 \%$ protease inhibitor cocktail). Western blot analysis protocols were previously described (20). Western blot analyses were performed with liver homogenates (30 $\mu \mathrm{g}$ protein) using anti-HO-1 antibody (1:2,000 dilution; 
Table I. Body weight and spleen weight in the sham and BDL groups at 2 weeks.

\begin{tabular}{lccc}
\hline & \multicolumn{2}{c}{2 weeks } \\
\cline { 2 - 4 } & Body weight $(\mathrm{g})$ & Spleen weight $(\mathrm{g})$ & Body weight $(\mathrm{g}) /$ Spleen weight $(\mathrm{g})(\%)$ \\
\hline Sham & $257 \pm 6$ & $0.96 \pm 0.07$ & $0.37 \pm 0.03$ \\
BDL & $219 \pm 12^{\mathrm{a}}$ & $1.49 \pm 0.19^{\mathrm{a}}$ & $0.68 \pm 0.04^{\mathrm{a}}$ \\
\hline
\end{tabular}

${ }^{\mathrm{a}} \mathrm{P}<0.01$ and ${ }^{\mathrm{b}} \mathrm{P}<0.05$ vs. sham group. BDL, bile duct ligation.

Table II. Serum biochemical index analysis in different groups (means $\pm \mathrm{SD}, \mathrm{n}=6$ /group).

\begin{tabular}{llcr}
\hline & $\begin{array}{c}\text { AST } \\
(\mathrm{U} / \mathrm{L})\end{array}$ & $\begin{array}{c}\text { ALT } \\
(\mathrm{U} / \mathrm{L})\end{array}$ & $\begin{array}{c}\text { TBIL } \\
(\mathrm{mg} / \mathrm{dl})\end{array}$ \\
\hline Sham & $139.00 \pm 4.52$ & $36.83 \pm 1.72$ & $0.83 \pm 0.23$ \\
BDL & $255.83 \pm 7.55^{\mathrm{a}}$ & $49.83 \pm 4.36^{\mathrm{a}}$ & $11.90 \pm 1.96^{\mathrm{a}}$ \\
CoPP & $406.67 \pm 14.81^{\mathrm{c}}$ & $72.00 \pm 3.52^{\mathrm{c}}$ & $13.75 \pm 2.23^{\mathrm{a}}$ \\
ZnPP & $216.00 \pm 7.54^{\mathrm{c}}$ & $37.17 \pm 2.48^{\mathrm{c}}$ & $5.53 \pm 2.23^{\mathrm{c}}$ \\
Fe & $309.83 \pm 13.61^{\mathrm{c}}$ & $43.83 \pm 4.96^{\mathrm{c}}$ & $13.82 \pm 1.94^{\mathrm{a}}$ \\
Splenectomy & $202.17 \pm 6.85^{\text {cf }}$ & $37.67 \pm 7.06^{\mathrm{c}}$ & $5.10 \pm 0.48^{\mathrm{c}}$ \\
\hline
\end{tabular}

${ }^{\mathrm{a}} \mathrm{P}<0.01$ and ${ }^{\mathrm{b}} \mathrm{P}<0.05$ vs. sham group; ${ }^{\mathrm{c}} \mathrm{P}<0.01$ and ${ }^{\mathrm{d}} \mathrm{P}<0.05$ vs. BDL group; ${ }^{\mathrm{e}} \mathrm{P}<0.01$ and ${ }^{\mathrm{f}} \mathrm{P}<0.05$ vs. ZnPP group. BDL, bile duct ligation; AST, aspartate aminotransferase; TBIL, total bilirubin; CoPP, cobalt protoporphyrin; ZnPP, zinc protoporphyrin.

Abcam), anti- $\beta$-actin antibody (1:500 dilution; Zhongshan Golden Bridge Biotechnology), and secondary anti-rabbit and anti-mouse IgG (1:500 dilution; Biosynthesis Biotechnology, Beijing, China). The intensity of each signal was corrected by the values obtained from the immunodetection of $\beta$-actin and the relative protein intensity expressed as fold of the content in the control group.

RNA isolation and gene expression analysis. Total RNA was extracted from livers following a standard guanidinium phenol-chloroform extraction protocol. The quantity of RNA was determined by measuring the optical density at $260 \mathrm{~nm}$ (A260 $\mathrm{nm}=1$ for $40 \mu \mathrm{g} / \mathrm{ml} \mathrm{RNA}$ ), and RNA purity was assessed by determining the A260/A280 nm ratio (pure RNA: A260/A280 nm $=2.0$ ) using a UV-1206 spectrophotometer (Shimadzu, Kyoto, Japan). An aliquot of each mixture was used for reverse transcription (RT)-PCR amplification using reagents purchased from Takara Bio, Inc. The primer sequences for HO-1 were 5'-ATATCTATACGGCCCTGGAA-3' (forward), 5'-GATGCTCGGGAAGGTGAA-3' (reverse) and the product size was $350 \mathrm{bp}$, while the primer sequences for $\beta$-actin were 5'-GAGGGAAATCGTGCGTGAC-3' (forward), 5'-CTGGAAGGTGGACAGTGAG-3' (reverse) and the product size was $445 \mathrm{bp}$. PCR products were separated by $2.5 \%$ agarose gel electrophoresis. The product bands were photographed and the density of each band was quantified. The results are expressed as the ratio of the band density for the target mRNA to that of $\beta$-actin mRNA.

Statistical analysis. All data are presented as the means \pm standard deviation. Statistical analysis was performed with SPSS software version 16.0 (IBM, Chicago, IL, USA). Groups were compared using one-way analysis of variance (ANOVA) with Dunnett's multiple comparison test (where applicable). Correlative comparison of two non-hierarchical variances with normal distribution was evaluated by Pearson's test, whereas the Spearman test was used for data with a non-normal distribution. P-values $<0.05$ were considered to indicate statistically significant differences.

\section{Results}

Splenectomy ameliorates liver fibroproliferation and decreases PVP. The common bile duct dilation, ascites, and jaundice were found in all BDL rats at four weeks post-operation, suggesting that BDL was successfully established. Two weeks after the operation, the spleens were much bigger and heavier in the BDL group relative to the sham group $(\mathrm{P}<0.01)$, and the body weight $(\mathrm{g}) /$ spleen weight $(\mathrm{g})$ ratio was larger in the BDL group $(\mathrm{P}<0.01)$ (Table I).

The serum levels of AST, ALT and TBIL in the BDL group were significantly higher than those in the sham group $(\mathrm{P}<0.01)$. Of note, they were at much lower levels in the splenectomy group than in the BDL group $(\mathrm{P}<0.01)$ (Table II). These data indicate that splenectomy improves liver function.

Hepatic fibrosis was evaluated by H\&E staining. The sham group showed normal architecture (Fig. 1Aa), whereas the BDL group exhibited inflammation, necrosis, and destruction of the lobular architecture (Fig. 1Ab). Compared with the BDL group, less fibrous hyperplasia and fibrosis extensions with fibroblast proliferation were noted in the splenectomy group (Fig. 1Af). The histopathological scores for liver fibrosis were higher 

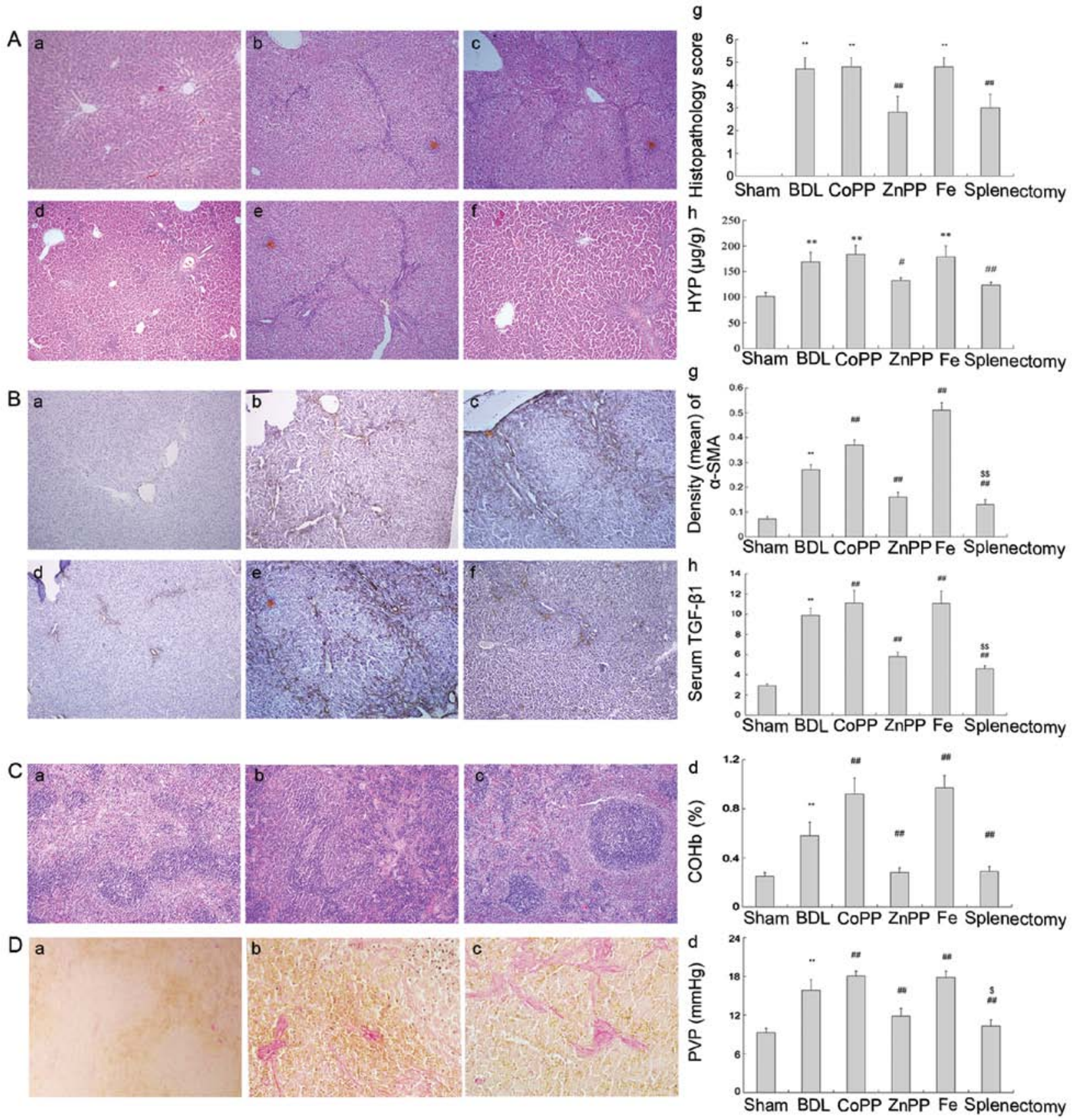

Figure 1. Assessment of liver fibrosis by different methods. (A) H\&E staining and HYP content. (a) Normal lobular architecture in the sham group; (b, $\mathrm{c}$ and e) fibrous hyperplasia in the BDL, CoPP and Fe groups; ( $\mathrm{d}$ and f) less fibrous hyperplasia in the ZnPP and splenectomy groups; ( $\mathrm{g}$ and $\mathrm{h}$ ) hepatic fibrosis was assessed using histopathological scores and HYP content (original magnification, x100). (B) Liver sections were stained with $\alpha$-SMA antibody. (a) There was only slight $\alpha$-SMA expression around central veins in the sham group. (b, c and e) Elevated expression was observed in the interlobular region in the BDL, CoPP and Fe groups. (d and f) There was less color in the ZnPP and splenectomy groups. The density (mean) of $\alpha$-SMA was measured by IPP (g) There was low $\alpha$-SMA expressed in the splenectomy and ZnPP groups compared with BDL $(\mathrm{P}<0.01)$. (h) Serum TGF- $\beta 1$ was detected by ELISA (original magnification, x100). (C) H\&E staining for spleen structure and COHb levels was measured. (a) Macrophages were distributed in the red pulp and marginal zone in normal spleen. (b and c) There were fewer macrophages in the red pulp at two and four weeks. (d) The levels of COHb were quantified (original magnification, $x 400$ ). (D) VG staining for collagen I in spleen and PVP measurement. (a) Little collagen I deposited in normal spleen, (b and c) but more accumulated in the spleen at two and four weeks. (d) PVP levels were measured (original magnification, $\mathrm{x} 400$ ). The data are represented as the means \pm SD. ${ }^{* *} \mathrm{P}<0.01,{ }^{*} \mathrm{P}<0.05$ vs. sham; ${ }^{\# /} \mathrm{P}<0.01,{ }^{\#} \mathrm{P}<0.05$ vs. BDL; ${ }^{\$ \$} \mathrm{P}<0.01,{ }^{\$} \mathrm{P}<0.05$ vs. ZnPP. a, Sham; b, BDL; c, CoPP; d, ZnPP; e, Fe; and f, splenectomy.

than for those that received a splenectomy (Fig. 1Ag). HYP, a product of collagen metabolism, is an amino acid characteristic of collagen. Compared with the BDL group, HYP content was appreciably lower following splenectomy $(\mathrm{P}<0.01)($ Fig. 1Ah).

Protein expression levels of $\alpha$-SMA and TGF- $\beta 1$ were significantly higher in the BDL group than in the sham group $(\mathrm{P}<0.01)$ (Fig. 1B). However, they were much lower in splenectomy-treated rats than in those receiving $\mathrm{BDL}(\mathrm{P}<0.01)$ (Fig. 1Bg). PVP levels were significantly higher in BDLthan in sham-treated rats $(\mathrm{P}<0.01)$. PVP levels were reduced following splenectomy, relative to BDL treatment $(\mathrm{P}<0.01)$ (Fig. 1Dd). These results suggest that splenectomy not only decreased PVP, but also improved liver function and reduced TGF- $\beta 1$ secretion, further decreasing hepatic fibrosis. 

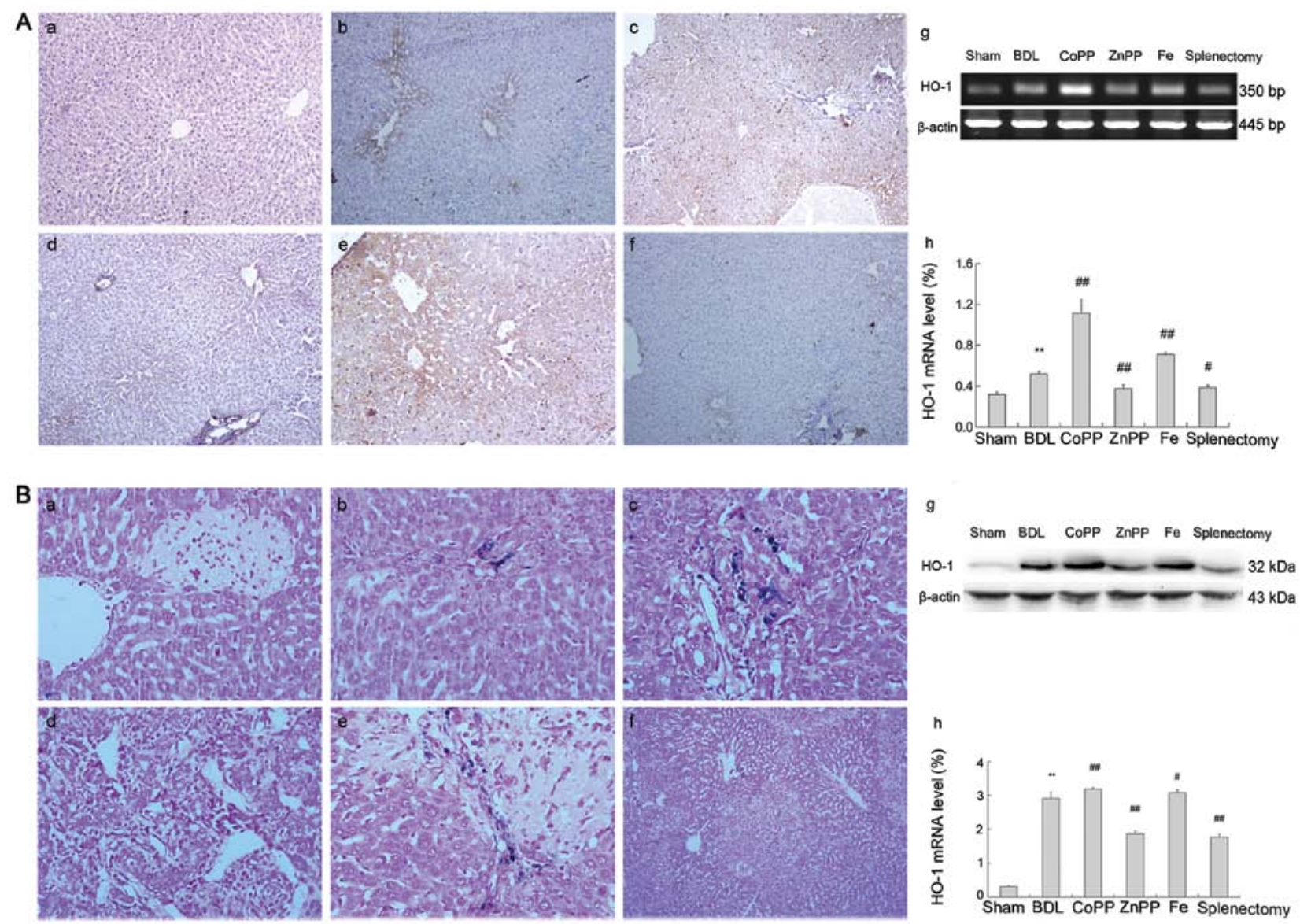

C a
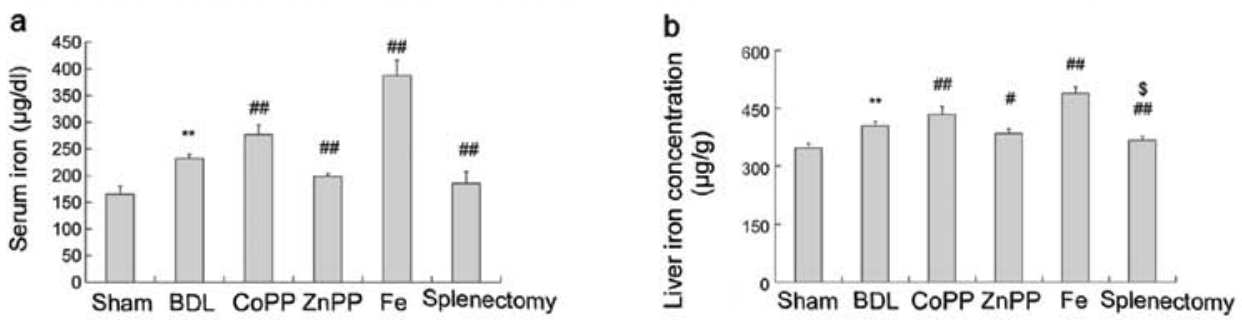

Figure 2. HO-1 and iron levels in liver. (A) Liver sections were stained with HO-1 antibody. Brown staining indicated immunopositivity. (a) HO-1 expression was minimal around central veins in the sham group. (b, $c$ and e) HO-1 was observed around central veins in the BDL, CoPP and Fe groups. (d and f) There was reduced staining in the ZnPP and splenectomy groups. ( $\mathrm{g}$ and $\mathrm{h}$ ) HO-1 mRNA increased in the BDL group relative to sham and were enhanced in the $\mathrm{CoPP}$ and Fe groups relative to the BDL group, and decreased in the ZnPP and DFX groups (original magnification, x400). (B) Perls' Prussian blue staining positive for iron was observed as blue stain. (a) No iron accumulated in the sham group. (b) A little iron accumulated mainly on Kupffer cells in the BDL group. (c) Substantially more iron accumulation was found in interlobular and macrophagocytes in the CoPP group. (d and f) Slight iron accumulation was detected in the $\mathrm{ZnPP}$ and splenectomy groups. (e) Substantial iron accumulation was present in the Fe group. ( $\mathrm{g}$ and $\mathrm{h}$ ) HO-1 protein increased in the BDL group relative to sham and were enhanced in the CoPP and Fe groups relative to the BDL group, and decreased in the ZnPP and DFX groups. (C) Serum and liver iron content. Iron content in both liver and serum increased more in the BDL than in the sham group. (a and b) Iron content decreased in the ZnPP and splenectomy groups compared with BDL, and enhanced Fe was observed in the CoPP and Fe groups. The data are represented as the means \pm SD. ${ }^{* *} \mathrm{P}<0.01$, "P<0.05 vs. sham; ${ }^{\# \#} \mathrm{P}<0.01,{ }^{\#} \mathrm{P}<0.05$ vs. BDL; ${ }^{\$ S} \mathrm{P}<0.01,{ }^{\text {SP }}<0.05$ vs. ZnPP. a, Sham; b, BDL; c, CoPP; d, ZnPP; e, Fe; and f, splenectomy.

Splenectomy reduces iron accumulation by inhibiting $\mathrm{HO}-1$ activity. The mRNA and protein expression levels of liver HO-1 increased significantly following BDL treatment compared to sham controls $(\mathrm{P}<0.01)$; they were lower in the splenectomy and $\mathrm{ZnPP}$ groups $(\mathrm{P}<0.01)$ than in the BDL group, but were elevated in the CoPP and Fe groups (Fig. 2Ag and h, Bg and h). Hepatic immunostaining showed that HO-1 was expressed mainly near centrilobular veins located on Kupffer cells, and partly in mesenchymal cells (Fig. 2Aa-f). Lower biochemical indicators, levels of $\alpha$-SMA and TGF- $\beta 1$ were lower in ZnPP-treated rats than in those receiving BDL treatment, yet higher in the CoPP and $\mathrm{Fe}$ groups. These results suggest that inhibiting HO-1 expression may improve liver function and decrease fibrosis. On the contrary, it aggravated liver injury (Fig. 1A and B).

Immunostaining of BDL spleen revealed multiple and patchy patterns of HO-1 (brown positive expression). Patterns were visible prominently in red pulp, while those in white pulp were few, if any (Fig. 3A-b). By contrast, the brown stain in the spleen collected from the CoPP and Fe-treated groups, and from macrophages in the red pulp, was markedly elevated. Cross-sections of red pulp appeared to be enlarged compared with analogous cross-sections from the BDL group, which decreased in splenectomy and ZnPP-treated rats (Fig. 3Aa-f). 

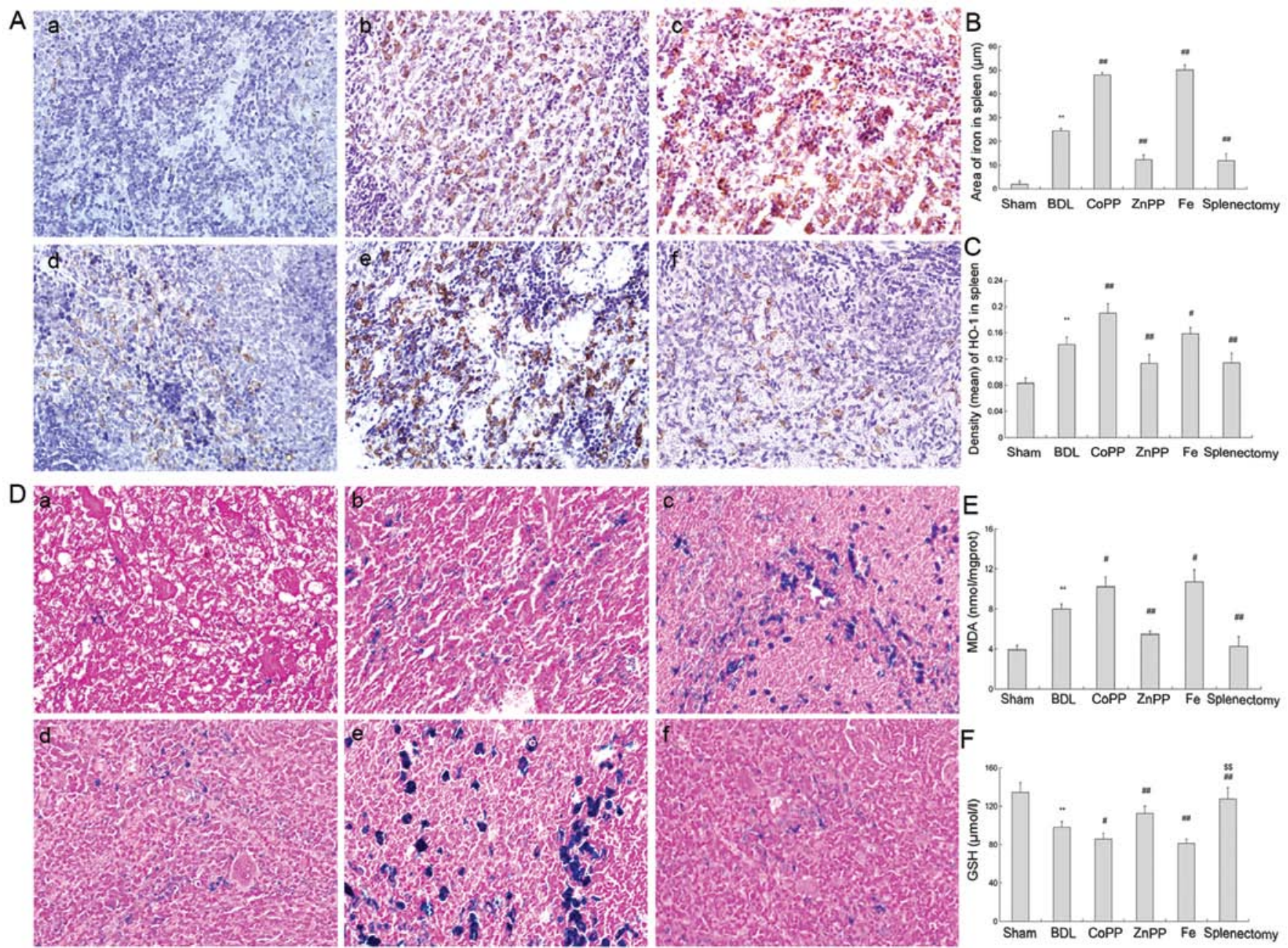

Figure 3. HO-1 and iron levels in the spleen. (A) Spleen sections were stained with HO-1 antibody. (a) Slight HO-1 expression was detected in the sham group. (b, $\mathrm{c}$ and e) There was more HO-1 expression in red pulp in the BDL, CoPP and Fe groups. ( $\mathrm{d}$ and $\mathrm{f}$ ) Compared with BDL rats, HO-1 expression decreased in the ZnPP and splenectomy groups. (B) Density (mean) of HO-1 in the spleen was measured by IPP, and was consistent with immunohistochemistry results $(\mathrm{P}<0.01)$. (C) Area of iron in spleen was detected by IPP. The iron levels were higher in BDL than in sham rats. Iron levels were decreased in the ZnPP and splenectomy groups compared with BDL, but enhanced in the CoPP and Fe groups $(\mathrm{P}<0.01)$. (D) Perls' Prussian blue staining in spleen. (a) Little iron accumulated in the sham group. (b, $c$ and e) Iron accumulation was found in the red pulp in BDL, CoPP and Fe groups. ( $\mathrm{d}$ and f) Compared with the BDL group, less iron was found in the ZnPP and splenectomy groups (original magnification, x400). (E) MDA levels in the liver were measured. MDA levels were markedly enhanced in $\mathrm{BDL}$ rats relative to sham rats. $\mathrm{HO}-1$ was increased in the CoPP and Fe groups relative to the BDL group, but decreased in the ZnPP and splenectomy groups ( $\mathrm{P}<0.01)$. (F) GSH levels were measured in the liver, and moved in opposition to MDA levels. The data are represented as the means $\pm \mathrm{SD}$. ${ }^{* *} \mathrm{P}<0.01,{ }^{*} \mathrm{P}<0.05 \mathrm{vs}$. sham; ${ }^{\# \#} \mathrm{P}<0.01,{ }^{\#} \mathrm{P}<0.05$ vs. BDL; ${ }^{\$ \$} \mathrm{P}<0.01,{ }^{\$} \mathrm{P}<0.05$ vs. ZnPP. a, Sham; b, BDL; c, CoPP; d, ZnPP; e, Fe; and f, spleen from the splenectomy group at two weeks.

Changes in HO-1 protein expression levels in the spleen were similar to those in the liver (Fig. 3B).

The iron serum levels in the BDL group were significantly higher than in sham controls $(\mathrm{P}<0.01)$, and were markedly lower following splenectomy and ZnPP treatments than after BDL treatment $(\mathrm{P}<0.01)$ (Fig. 2Ca). Also, the change of liver iron content correlated with the change of serum iron levels (Fig. 2Cb). We used Prussian blue stain to localize iron accumulation in hepatic and splenic tissue. In the liver and spleen, iron accumulated in Kupffer cells and macrophages in the BDL, CoPP and Fe groups (Figs. 2B and 3D). However, iron staining was essentially absent in the sham, splenectomy, and $\mathrm{ZnPP}$ treated groups (Fig. 2Ba, $\mathrm{d}$ and f). The areas of iron accumulation in the spleen are shown in Fig. 3C.

These results indicate that the spleen plays an important role in HO-1 expression and iron accumulation, and that splenectomy reduces liver HO-1 expression and reduces iron accumulation. By contrast, enhanced HO-1 expression led to increased accumulation of hepatic iron.

The HO/CO pathway is involved in regulating PVP. Splenic structure was evaluated by H\&E staining (Fig. 1C). In normal spleen, macrophages were distributed in red pulp and marginal zones, but were less prevalent in BDL spleen (Fig. 1Ca-c). Collagen deposits were also detected in the spleen. More collagen fibers were found around the splenic corpuscle in the 4th week following BDL treatment than in the 2nd week (Fig. 1Da-c). This may be one factor regulating PVP formation in cirrhotic rats.

The $\mathrm{COHb}$ level in arterial blood was significantly higher in the BDL group than in the sham group $(\mathrm{P}<0.01)$, significantly lower following splenectomy and ZnPP treatment, and higher in the CoPP and $\mathrm{Fe}$-treated groups than in the BDL group $(\mathrm{P}<0.01)$ (Fig. 1Dd). PVP was significantly higher in BDL than in sham 


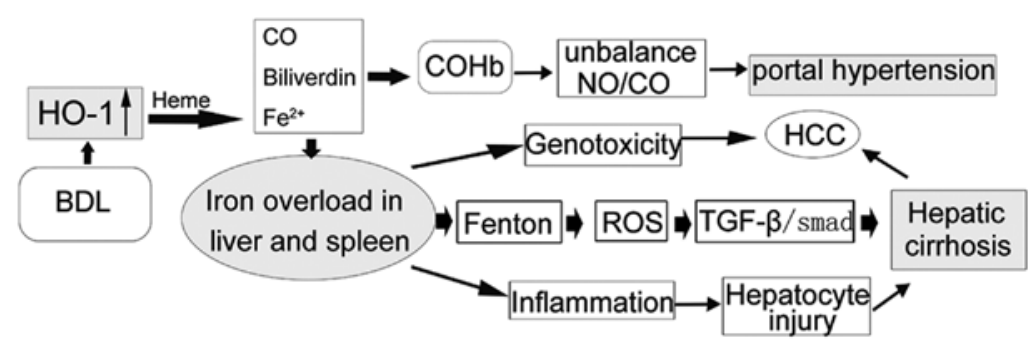

Figure 4. HO-1 regulates iron homeostasis and portal vein pressure in BDL rats. Inducing HO-1 expression mediates iron accumulation, which accelerates oxidative stress and further activates the TGF $\beta 1 /$ Smad pathway, aggravating liver cirrhosis. Excessive iron accumulation stimulates inflammation by the Fenton reaction and injures hepatocytes, leading to hepatocellular carcinoma due to increased genotoxicity. Splenectomy reduces iron overload and decreases PVP by inhibiting HO-1 activity, further alleviating liver cirrhosis.

$(\mathrm{P}<0.01)$. Compared with BDL, PVP decreased following splenectomy and $\mathrm{ZnPP}$ treatment $(\mathrm{P}<0.01)$, and was enhanced in $\mathrm{CoPP}$ and $\mathrm{Fe}$ rats $(\mathrm{P}<0.01)$ (Fig. 1Dd). Moreover, PVP decreased following splenectomy relative to $\mathrm{ZnPP}$ treatment $(\mathrm{P}<0.05)$. We hypothesized that splenectomy may decrease $\mathrm{COHb}$ levels and regulate $\mathrm{PVP}$ through the $\mathrm{HO} / \mathrm{CO}$ pathway.

Splenectomy reduces oxidative stress partly through the $\mathrm{HO} / \mathrm{CO}$ pathway. MDA levels increased significantly following BDL compared to sham controls, and GSH levels were reduced $(\mathrm{P}<0.01)$ (Fig. 3E and F). Splenectomy resulted in a lower level of MDA and elevated GSH levels relative to BDL $(\mathrm{P}<0.01)$ (Fig. 3E). This suggests that oxidative stress was activated in cirrhotic rats.

The level of MDA was slightly higher in the CoPP and Fe-treated groups than in the BDL group $(\mathrm{P}<0.05)$, but lower in ZnPP-treated rats. GSH levels were significantly enhanced by $\mathrm{ZnPP}$ treatment relative to $\mathrm{BDL}$ treatment $(\mathrm{P}<0.01)$, but decreased following CoPP and Fe treatment (Fig. 3F). Moreover, GSH levels were elevated following splenectomy compared to ZnPP treatment $(\mathrm{P}<0.01)$. Markedly, inhibiting HO-1 expression reduced oxidative stress, and induced HO-1 expression and iron accumulation, leading to liver injury.

Correlation analysis revealed that both SOD and MDA were significantly correlated with HYP levels $(R=-0.838$, 0.871 , respectively, $\mathrm{P}<0.01)$. These data show that oxidative stress may lead to extracellular matrix (ECM) deposition in the liver and aggravate hepatic fibrosis. These data further indicate that reducing oxidative stress may lighten hepatic fibrosis, partly through the $\mathrm{HO} / \mathrm{CO}$ pathway after splenectomy.

\section{Discussion}

Splenectomy is an effective operation for decreasing PVP. In this study, we found that splenectomy also influences the cirrhotic rat liver by altering HO-1 expression, resulting in iron homeostasis. Two weeks after splenectomy, the levels of AST, ALT and TBIL decreased, as did the serum concentrations of TGF- $\beta 1, \alpha$-SMA and HYP in the liver. Recent studies suggest that splenectomy may cause anti-inflammatory effects in the portal system in addition to decreasing PVP and reversing hypersplenism, which improves hepatic function $(4,5)$. Moreover, splenectomy may moderate fibrosis by decreasing TGF- $\beta 1$ secretion. Spleen-derived TGF- $\beta 1$ plays an inhibitory role in healing hepatic cirrhosis by prohibiting regeneration of damaged liver $(21,22)$. We found it also improved liver function and decreased liver fibrosis following BDL, although splenectomy was initially performed to decrease PVP.

The majority of endogenous $\mathrm{CO}$ is catalyzed by inducible expression of HO-1. The release of $\mathrm{CO}$ by vascular cells may modulate blood flow and maintain the integrity of the vessel wall (23). It has been suggested that CO interacts with $\mathrm{NO}$, which is a potent activator of soluble guanylate cyclase and a vasodilator $(24,25)$. Indeed, excessive CO production, a consequence of HO-1 overexpression, could play a critical role in modulating vascular tone under different pathological situations (26). $\mathrm{COHb}$ levels can be used to estimate $\mathrm{HO}$ activity in experimental animals (27). Notably, we observed that upregulated $\mathrm{COHb}$ resulted from increased $\mathrm{HO}-1$, which aggravated PVP in BDL rats. Moreover, splenectomy and lower levels of $\mathrm{COHb}$ can decrease PVP, with similar results found in the $\mathrm{ZnPP}$ treatment group. We reasoned that $\mathrm{CO}$ may decrease PVP in early stages, and excessive $\mathrm{CO}$ may be harmful by reducing PVP, leading to an unbalanced $\mathrm{NO} / \mathrm{CO}$ system in the last stage of hepatic fibrosis. It therefore seems appropriate to reduce PVP by decreasing CO.

Our previous study indicated that HO-1 induction can ameliorate immune liver fibrosis (28). Upregulation of HO-1 interferes with chronic inflammation and prevents progression of liver fibrosis in Mdr2 knockout mice (29). Our study found that inhibition of HO-1 could modulate BDL-induced liver fibrosis, which was not in accordance with previous results. We hypothesized that HO-1 plays different roles in the progression of liver fibrosis. In early stage liver fibrosis, inducing HO-1 may have a protective action, but aggravates liver function and PVP during end stage cirrhosis with portal hypertension. Moreover, fibrosis resulting from different animal models may offer another explanation for these results.

We investigated the relationship between HO-1 expression and iron accumulation by inhibiting or inducing HO-1 expression. Inhibiting HO-1 activity reduced iron accumulation in the liver and spleen, and further attenuated hepatic fibrosis. Splenectomy produced similar results, which may be due, partly, to reduced HO-1 expression. Khan et al (30) reported that an increase in HO-1 expression is associated with iron accumulation, and $\mathrm{HO}-1$ activity contributes to increased levels of intracellular labile iron (31). HO-1 gene expression is upregulated by iron, suggesting that degradation of internalized heme may be controlled by a positive feedback loop (32). In the present study, HO-1 was upregulated 
following Fe treatment. However, anemia and iron accumulation in the kidney and liver are found in HO-1-deficient mice (33). Absent HO-1 and other HO subtypes, such as HO-2 and HO-3, may play a major role in iron homeostasis. We considered that HO-1 was necessary to form iron homeostasis, and inhibiting it, but not knocking it out, may be useful to reduce iron accumulation.

It has previously been shown that increased deposition of iron in the liver often triggers oxidative stress and inflammation and induces liver cell damage (34). Iron participates in the Fenton and Haber-Weiss reaction, and excessive redox-active iron leads to oxidative stress, with damage to membranes, proteins, and DNA (35). Nontransferrin-bound iron plays a key role in iron overload in severe cirrhosis (36). Our data show that iron accumulation increases oxidative stress and aggravates liver injury in CoPP and Fe-treated groups. This demonstrates that iron may play a pivotal role in hepatic fibrosis.

In our study, iron levels in serum, liver, and spleen all decreased in the splenectomy-treated animals, which showed lower HO-1 than the BDL group. Similar results were found in the ZnPP group. A previous study demonstrated that HO-1 activity is found in the spleen (37). We found enhanced HO-1 expression in the liver and spleen at two and four weeks following BDL. Spleen is a source of HO-1 production and splenectomy could therefore decrease HO-1 generation.

Previous studies have shown that spleen volume negatively correlates with red blood cell and platelet counting (38). Others have noted that splenic hypertrophy partly explains the anemia process, and splenomegaly is widely observed in human fascioliasis (39). These reports indicate that splenomegaly is a key reason for RBC damage and iron production from heme in cirrhotic patients. Splenectomy decreased red blood cell damage and further reduced iron production from heme degradation. This may be a benefit for cirrhotic patients with splenomegaly.

Reactive oxygen species (ROS) may represent a relevant profibrogenic stimulus for hepatic stellate cells (HSCs), promote production of type I collagen, and act as an intracellular signaling mediator of TGF- $\beta(40,41)$. Splenectomy reduced oxidative stress in BDL rats and further decreased collagen deposition. Of note, however, similar results were found in the $\mathrm{ZnPP}$ group due to inhibition of HO-1 expression. HO-1 protected hepatocytes from ethanol-derived oxidative stress via the MAPK/Nrf2 pathway, in primary human hepatocytes (42). Iron accumulation increases liver injury through oxidative stress in nutritional steatohepatitis (43). We thus reasoned that the influences of pro-oxidant activities resulted from iron accumulation and were more effective than the antioxidant effects mediated by HO-1.

In conclusion, splenectomy decreases PVP. Moreover, we found it improves liver function partly through the $\mathrm{HO} / \mathrm{CO}$ pathway during the hepatic cirrhotic process in rats. Inhibition of HO-1 expression by splenectomy led to reduced iron production (Fig. 4). However, we lacked a large-scale clinical trial and in vitro study to better clarify the exact role of the $\mathrm{HO} / \mathrm{CO}$ pathway in cirrhotic patients following splenectomy, as it is difficult to obtain samples from patients who recover after surgery and who volunteer for a liver biopsy. In addition, we have reason to believe that a reduction in $\mathrm{CO}$ due to $\mathrm{HO}-1$ inhibition may be a novel therapeutic option for decreasing PVP. The HO/CO pathway may play a pivotal role in patients with splenectomy to intervene in liver cirrhosis and further reduce PVP.

\section{Acknowledgements}

This study was supported by the National Natural Science Foundation of China, no. 30970886, the Technology Project of Dalian, no. 2008E13SF193 and the Doctoral Initial Funding of Liaoning Province, no. 20121110.

\section{References}

1. Imura S, Shimada M, Utsunomiya T, et al: Impact of splenectomy in patients with liver cirrhosis: Results from 18 patients in a single center experience. Hepatol Res 40: 894-900, 2010.

2. Ito K, Ozasa H, Noda Y and Horikawa S: Splenic artery ligation ameliorates hepatic ischemia and reperfusion injury in rats. Liver Int 26: 254-260, 2006

3. Ito K, Ozasa H and Horikawa S: Effects of prior splenectomy on remnant liver after partial hepatectomy with Pringle maneuver in rats. Liver Int 25: 438-444, 2005.

4. Shimada M, Ijichi H, Yonemura Y, et al: The impact of splenectomy or splenic artery ligation on the outcome of a living donor adult liver transplantation using a left lobe graft. Hepatogastroenterology 51: 625-629, 2004.

5. Chen XP, Wu ZD, Huang ZY and Qiu FZ: Use of hepatectomy and splenectomy to treat hepatocellular carcinoma with cirrhotic hypersplenism. Br J Surg 92: 334-339, 2005.

6. Uehara H, Akahoshi T, Kawanaka H, et al: Endothelin-1 derived from spleen-activated Rho-kinase pathway in rats with secondary biliary cirrhosis. Hepatol Res 42: 1039-1047, 2012.

7. Arakawa Y, Shimada M, Uchiyama $\mathrm{H}$, et al: Beneficial effects of splenectomy on massive hepatectomy model in rats. Hepatol Res 39: 391-397, 2009.

8. Ito K, Ozasa H, Yoneya R and Horikawa S: Splenectomy ameliorates hepatic ischemia and reperfusion injury mediated by heme oxygenase-1 induction in the rat. Liver 22: 467-473, 2002.

9. Tarquini R, Masini E, La Villa G, et al: Increased plasma carbon monoxide in patients with viral cirrhosis and hyperdynamic circulation. Am J Gastroenterol 104: 891-897, 2009.

10. Duan ZJ, Yang D, Wang F, Sun YJ, Sun XY and Zheng ML: Heme oxygenase-1 regulates the major route involved in formation of immune hepatic fibrosis in rats. Chin Med J 123: 3304-3308, 2010.

11. Sass G, Barikbin R and Tiegs G: The multiple functions of heme oxygenase-1 in the liver. Z Gastroenterol 50: 34-40, 2012.

12. Naik JS, O'Donaughy TL and Walker BR: Endogenous carbon monoxide is an endothelial-derived vasodilator factor in the mesenteric circulation. Am J Physiol Heart Circ Physiol 284: H838-H845, 2003.

13. Guo SB, Duan ZJ, Li Q and Sun XY: Effect of heme oxygenase-1 on renal function in rats with liver cirrhosis. World J Gastroenterol 17: 322-328, 2011.

14. Guo SB, Duan ZJ, Li Q and Sun XY: Effects of heme oxygenase-1 on pulmonary function and structure in rats with liver cirrhosis. Chin Med J 124: 918-922, 2011.

15. Iwamoto T, Terai S, Mizunaga Y, et al: Splenectomy enhances the anti-fibrotic effect of bone marrow cell infusion and improves liver function in cirrhotic mice and patients. J Gastroenterol 47: 300-312, 2012.

16. Brown KE, Dennery PA, Ridnour LA, et al: Effect of iron overload and dietary fat on indices of oxidative stress and hepatic fibrogenesis in rats. Liver Int 23: 232-242, 2003.

17. Fallon MB, Abrams GA, McGrath JW, Hou Z and Luo B: Common bile duct ligation in the rat: a model of intrapulmonary vasodilatation and hepatopulmonary syndrome. Am J Physiol 272: G779-G784, 1997.

18. Luo B, Abrams GA and Fallon MB: Endothelin-1 in the rat bile duct ligation model of hepatopulmonary syndrome: correlation with pulmonary dysfunction. J Hepatol 29: 571-578, 1998.

19. Shackelford C, Long G, Wolf J, Okerberg C and Herbert R: Qualitative and quantitative analysis of nonneoplastic lesions in toxicology studies. Toxicol Pathol 30: 93-96, 2002.

20. Kakinuma S, Tanaka Y, Chinzei R, et al: Human umbilical cord blood as a source of transplantable hepatic progenitor cells. Stem Cells 21: 217-227, 2003. 
21. Ueda S, Yamanoi A, Hishikawa Y, Dhar DK, Tachibana M and Nagasue N: Transforming growth factor-betal released from the spleen exerts a growth inhibitory effect on liver regeneration in rats. Lab Invest 83: 1595-1603, 2003.

22. Akahoshi T, Hashizume M, Tanoue K, et al: Role of the spleen in liver fibrosis in rats may be mediated by transforming growth factor beta-1. J Gastroenterol Hepatol 17: 59-65, 2002.

23. Giles TD, Sander GE, Nossaman BD and Kadowitz PJ: Impaired vasodilation in the pathogenesis of hypertension: focus on nitric oxide, endothelial-derived hyperpolarizing factors, and prostaglandins. J Clin Hypertens 14: 198-205, 2012.

24. Johnson RA, Kozma F and Colombari E: Carbon monoxide: from toxin to endogenous modulator of cardiovascular functions. Braz J Med Biol Res 32: 1-14, 1999.

25. Furchgott RF and Jothianandan D: Endothelium-dependent and -independent vasodilation involving cyclic GMP: relaxation induced by nitric oxide, carbon monoxide and light. Blood Vessels 28: 52-61, 1991.

26. Decaluwe K, Pauwels B, Verpoest S and Van de Voorde J: Divergent mechanisms involved in CO and CORM-2 induced vasorelaxation. Eur J Pharmacol 674: 370-377, 2012.

27. Carter EP, Hartsfield CL, Miyazono M, Jakkula M, Morris KG Jr and McMurtry IF: Regulation of heme oxygenase-1 by nitric oxide during hepatopulmonary syndrome. Am J Physiol Lung Cell Mol Physiol 283: L346-L353, 2002.

28. Wang F, Duan ZJ and Sun YJ: Influence of heme oxygenase-1 expression on immune liver fibrosis induced by cobalt protoporphyrin in rats. World J Gastroenterol 15: 3009-3014, 2009.

29. Barikbin R, Neureiter D, Wirth J, et al: Induction of heme oxygenase 1 prevents progression of liver fibrosis in Mdr2 knockout mice. Hepatology 55: 553-562, 2012.

30. Khan ZA, Barbin YP, Cukiernik M, Adams PC and Chakrabarti S Heme-oxygenase-mediated iron accumulation in the liver. Can J Physiol Pharmacol 82: 448-456, 2004.

31. Kartikasari AE, Wagener FA, Yachie A, Wiegerinck ET, Kemna EH and Swinkels DW: Hepcidin suppression and defective iron recycling account for dysregulation of iron homeostasis in heme oxygenase-1 deficiency. J Cell Mol Med 13: 3091-3102, 2009.
32. Crichton RR, Wilmet S, Legssyer R and Ward RJ: Molecular and cellular mechanisms of iron homeostasis and toxicity in mammalian cells. J Inorg Biochem 91: 9-18, 2002.

33. Immenschuh S, Baumgart-Vogt $E$ and Mueller S: Heme oxygenase-1 and iron in liver inflammation: a complex alliance. Curr Drug Targets 11: 1541-1550, 2010.

34. Ramm GA and Ruddell RG: Iron homeostasis, hepatocellular injury, and fibrogenesis in hemochromatosis: the role of inflammation in a noninflammatory liver disease. Semin Liver Dis 30: 271-287, 2010.

35. Pietrangelo A: Metals, oxidative stress, and hepatic fibrogenesis Semin Liver Dis 16: 13-30, 1996.

36. Deugnier Y and Turlin B: Pathology of hepatic iron overload. Semin Liver Dis 31: 260-271, 2011.

37. Chen YC, Gines P, Yang J, et al: Increased vascular heme oxygenase-1 expression contributes to arterial vasodilation in experimental cirrhosis in rats. Hepatology 39: 1075-1087, 2004.

38. Shi BM, Wang XY, Mu QL, Wu TH and Xu J: Value of portal hemodynamics and hypersplenism in cirrhosis staging. World $\mathrm{J}$ Gastroenterol 11: 708-711, 2005.

39. Valero MA, Girones N, Garcia-Bodelon MA, et al: Anaemia in advanced chronic fasciolosis. Acta Trop 108: 35-43, 2008.

40. Rhyu DY, Park J, Sharma BR and Ha H: Role of reactive oxygen species in transforming growth factor-beta1-induced extracellular matrix accumulation in renal tubular epithelial cells. Transplant Proc 44: 625-628, 2012.

41. Urtasun R, Conde de la Rosa L and Nieto N: Oxidative and nitrosative stress and fibrogenic response. Clin Liver Dis 12: 769-790, viii, 2008.

42. Yao P, Nussler A, Liu L, et al: Quercetin protects human hepatocytes from ethanol-derived oxidative stress by inducing heme oxygenase-1 via the MAPK/Nrf2 pathways. J Hepatol 47: 253-261, 2007.

43. Okada K, Warabi E, Sugimoto H, et al: Nrf2 inhibits hepatic iron accumulation and counteracts oxidative stress-induced liver injury in nutritional steatohepatitis. J Gastroenterol 47: 924-935, 2012 . 